\title{
Pyogenic granuloma on the upper lip: an unusual location
}

\author{
Eduardo Sanches GONÇALES'1 , José Humberto DAMANTE², Cassia Maria FISCHER RUBIRA, \\ Luís Antônio de Assis TAVEIRA ${ }^{4}$ \\ 1- DDS, PhD, Assistant Professor, Department of Stomatology, Bauru School of Dentistry, University of São Paulo, Bauru, SP, Brazil. \\ 2- DDS, PhD, Professor, Department of Stomatology, Bauru School of Dentistry, University of São Paulo, Bauru, SP, Brazil. \\ 3- DDS, PhD in Stomatology, Bauru School of Dentistry, University of São Paulo, Bauru, SP, Brazil. \\ 4- DDS, PhD, Associate Professor, Department of Stomatology, Bauru School of Dentistry, University of São Paulo, Bauru, SP, Brazil.
}

Corresponding address: Eduardo Sanches Gonçales - Faculdade de Odontologia de Bauru - USP - Departmento de Estomatologia - Al. Dr. Octavio Pinheiro Brisolla 9-75 - 17.012-901 - Bauru, SP - Brasil - Phone: 551432358250 - e-mail:eduardogoncales@usp.br

Received: March 23, 2009 - Modification: September 16, 2009 - Accepted: October 11, 2009

\section{ABSTRACT}

\begin{abstract}
Dyogenic granuloma (PG) is a benign non-neoplastic mucocutaneous lesion. It is a reactional response to constant minor trauma and might be related to hormonal changes. In the mouth, PG is manifested as a sessile or pedunculated, resilient, erythematous, exophytic and painful papule or nodule with a smooth or lobulated surface that bleeds easily. PG preferentially affects the gingiva, but may also occur on the lips, tongue, oral mucosa and palate. The most common treatment is surgical excision. This paper describes a mucocutaneous PG on the upper lip, analyzing the clinical characteristics and discussing the features that distinguish this lesion from other similar oral mucosa lesions. The diagnosis of oral lesions is complex and leads the dentist to consider distinct lesions with different diagnostic methods. This case report with a 4 year-follow-up calls the attention to the uncommon mucocutaneous labial location of PG and to the fact that surgical excision is the safest method for diagnosis and treatment of PG of the lip, even when involving the mucosa and skin
\end{abstract}

Key words: Pyogenic granuloma. Pregnancy tumor. Chemically induced.

\section{INTRODUTION}

Pyogenic granuloma (PG) is a benign nonneoplastic mucocutaneous lesion, with the term "pyogenic" being used erroneously since this condition does not produce purulent secretion ${ }^{1}$ and is not related to infection? ${ }^{7}$. PG is also known as pregnancy granuloma or pregnancy tumor when occurring in pregnant women, or as vascular epulis, benign vascular tumor and hemangiomatous granuloma ${ }^{4}$.

Jafarzadeh, et al.7 (2006) defined PG as an inflammatory overgrowth of the oral mucosa caused by minor trauma or irritation. According to Neville, et al. ${ }^{9}$ (1998), these injuries might be caused in the mouth by gingival inflammation due to poor hygiene, trauma or local infection. Lin and Janniger $^{8}$ (2004) reported an association between dermatosis and the occurrence of PG.

PG is manifested as a sessile or pedunculated, resilient, erythematous, exophytic and painful papule or nodule with a smooth or lobulated surface that bleeds easily ${ }^{4,7}$. The gingiva is the most commonly affected site by PGs, accounting for $75 \%$ of all cases ${ }^{8}$, although occurrence of these lesions on the lips, tongue, oral mucosa, palate $^{9}$ and fingers ${ }^{2}$ has also been reported. PGs affecting the labial mucosa are rare ${ }^{4}$.

A higher frequency of $P G$ is observed in the second decade of life ${ }^{1}$, especially among women, probably because of the vascular effects of female hormones. ${ }^{8}$ A preference for children has been reported by some investigators ${ }^{7}$.

Microscopically, PG is characterized by marked vascular proliferation amidst granulation tissue and chronic inflammatory infiltrate. When ulcerated, the surface of the lesion is covered with fibrin. Older lesions may present areas of fibrosis ${ }^{10}$.

In view of its clinical characteristics, the differential diagnosis of PG includes peripheral giant cell granuloma, peripheral ossifying fibroma, metastases of malignant tumors, hemangioma, conventional granulation tissue, fistula, inflammatory gingival hyperplasia, Kaposi's sarcoma, angiosarcoma, non-Hodgkin's lymphoma' and cutaneous horn in the lower lip ${ }^{11}$. The final diagnosis depends on biopsy?

Surgical excision is the most common 
treatment ${ }^{12}$; however, this may result in scars $^{5}$, which is why the use of more conservative treatments, such as cryosurgery ${ }^{6,14}$ and laser surgery, is preferred ${ }^{3,5}$. The recurrence is about $3 \%$ after simple excision ${ }^{13}$.

This is a case report of PG on the upper lip. It presents the clinical characteristics and discusses the features that distinguish this lesion from other similar lesions of the oral mucosa, with special emphasis on the diagnosis and treatment of this condition.

\section{CASE REPORT}

A 12-year-old mulatto girl presented a 3-month history of a "blister on the upper lip". The initial aspect of the blister changed after application of thinner because the lesion bled easily. The lesion was asymptomatic, grew slowly and continued to bleed. The patient's main complaint was her fear of going to school because of the cruel comments by other students. Medical history revealed no other systemic or local contributory factors associated with the oral finding. No palpable cervical lymph nodes were detected.

Clinical examination revealed a reddish-yellow, ulcerated, superficial nodule measuring $1 \mathrm{~cm}$ in diameter, involving the mucocutaneous line of the upper lip on the left, which bled on touch and was resilient to palpation (Figure 1). The diagnostic hypotheses were PG, recent oral syphilis (hard chancre) or tuberculous ulcer. Complementary blood tests, complete blood count, erythrocyte sedimentation rate, VDRL (venereal disease research laboratory) test and radiography of the pulmonary fields made it possible to exclude infectious diseases, a fact also supported by the patient's general good health.

The nodule was removed by excisional biopsy (Figure 2). Microscopic analysis showed an oral mucosa consisting of continuous, parakeratinized, stratified pavement epithelium covered with

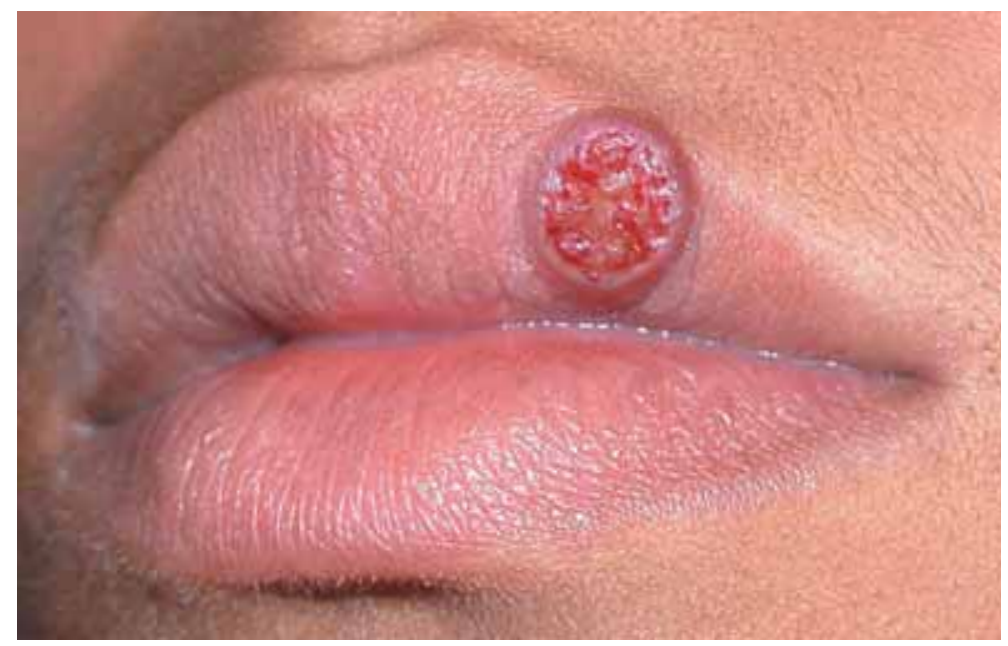

Figure 1- Reddish-yellow, ulcerated, superficial nodule involving the mucocutaneous line of the upper lip

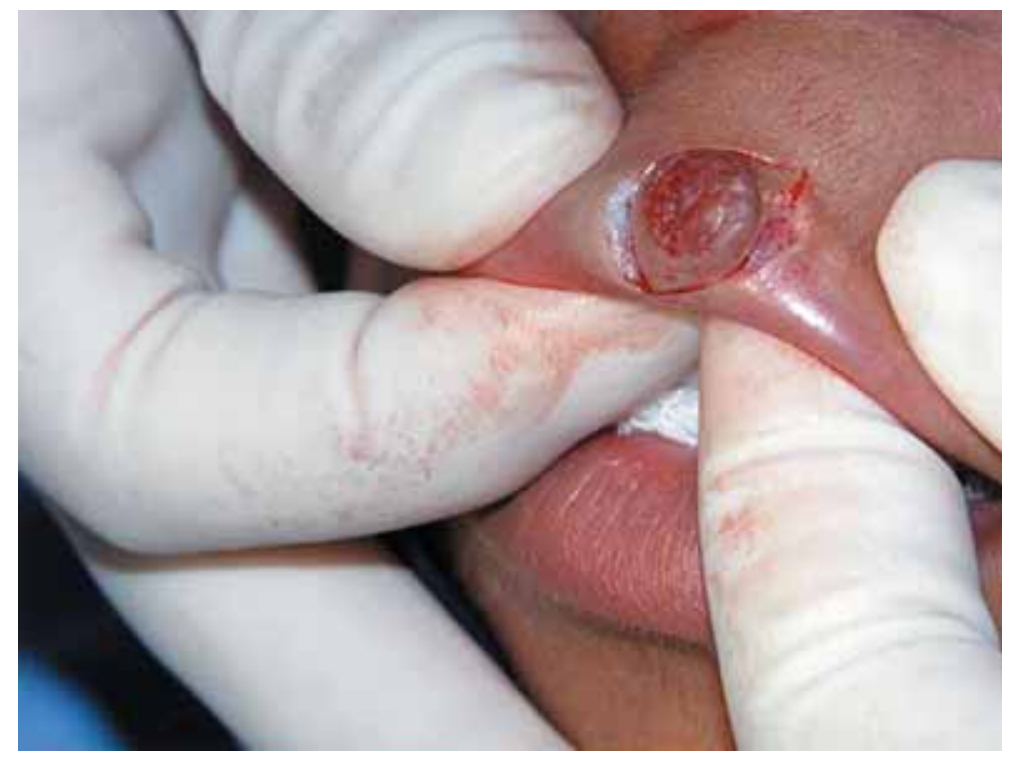

Figure 2- Excisional biopsy to remove the nodule 
a serofibrinous membrane. The underlying granulation tissue was rich in blood vessels, fibroblasts and macrophages. Skeletal striated muscle fibers were observed in the deep layers (Figure 3). The diagnosis was PG. After 6 months (Figure $4 \mathrm{~A}$ ) of follow-up the patient was satisfied

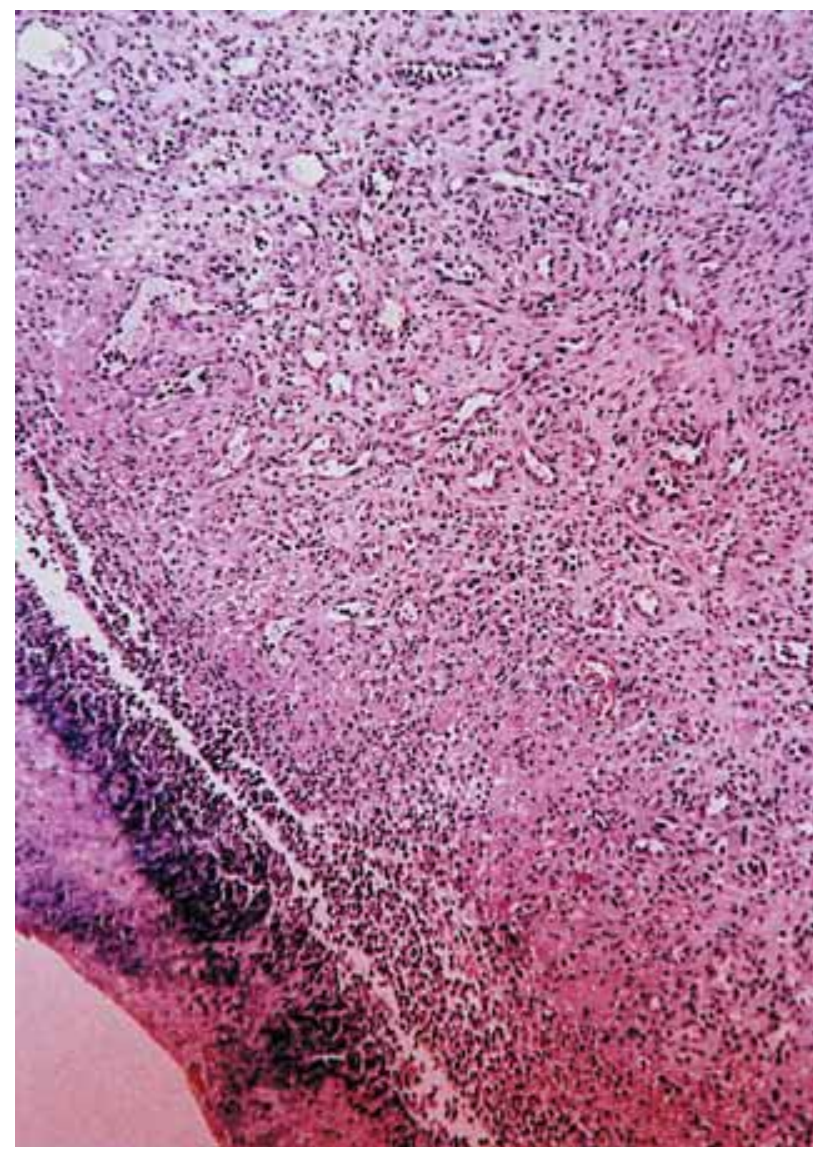

Figure 3- Microscopic aspect showing an oral mucosa consisting of continuous parakeratinized, stratified pavement epithelium covered with a serofibrinous membrane. The underlying granulation tissue was rich in blood vessels, fibroblasts and macrophages. Skeletal striated muscle fibers were observed in the deep layers (Original magnification $\times$ 25) with the esthetic outcome, and 4 years (Figure $4 \mathrm{~B}$ ) after surgery the patient was definitively discharged.

\section{DISCUSSION}

There is consensus that PG is a reactional lesion formed in response to minor trauma and/or chronic irritation, with reports of its occurrence after lowintensity traumatic injuries ${ }^{1,2,4,8-10}$.

PGs are preferentially found in the gingiva ${ }^{9}$, whereas its occurrence in the upper lip seems to be rare ${ }^{5}$. Thus, the present case of PG affecting the upper lip, a region rarely subjected to trauma, is unusual, especially the location of the lesion in the lip vermilion (with involvement of the mucocutaneous line and skin). This unusual location led to other diagnostic hypotheses for ulcerated lesions of the upper lip, such as hard chancre (lesion resulting from inoculation of Treponema pallidum) and labial tuberculous ulcer.

Although Jafarzadeh, et al. ${ }^{7}$ (2006) have suggested that the differential diagnosis of PG should include a large number of lesions such as malignant ulcers, sarcoidosis, syphilis, aphthous ulcers, mycotic infection and traumatic injuries. Neville, et al. $^{9}$ (1998) suggested the inclusion of tuberculous oral lesions in the differential diagnosis since, although uncommon, these lesions may present nodular, granular or ulcerated areas.

Treponema pallidum is the etiological agent of syphilis, a chronic, sexually transmitted disease of universal incidence that can affect various organs and systems, including the mouth, which is one of the main extragenital sites of syphilis. Oral primary syphilis manifests as a deep solitary ulcer with a red or brown base and irregular border usually located on the upper lip. The disease is followed by cervical lymphadenopathy, which is observed 1 to 3 weeks after acquisition of the infection. The condition might be confused with other solitary ulcerated lesions, especially traumatic lesions. Diagnosis of primary syphilis is made analyzing

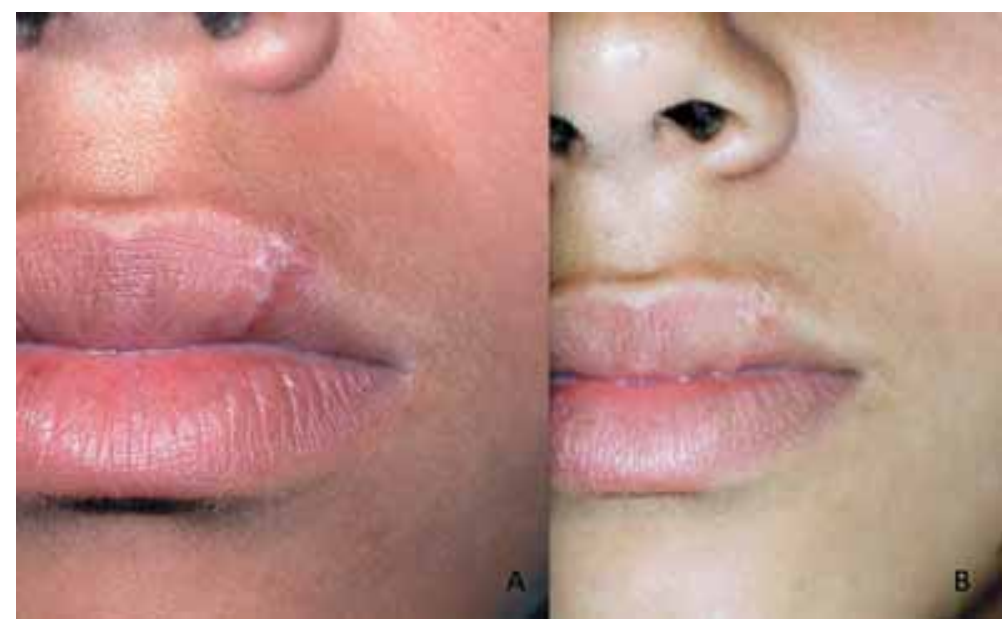

Figure 4- Labial aspect 6 months (A) and 4 years (B) postoperatively 
the patient's social and sexual lifestyle and his/her partner, together with nonspecific serological tests, such as the VDRL test, fluorescent treponemal antibody and treponemal immobilization ${ }^{9,10,12}$.

Tuberculosis is also a universal disease whose understanding is of utmost importance for the Brazilian population because of the lack of basic sanitation and adequate sanitary conditions. Mycobacterium tuberculosis, the causal agent of tuberculosis, is contracted through sputum or air, reaching the lung and causing primary infection. The primary complex then heals through fibrosis and calcification, which are visible on chest x-rays. Tuberculosis can also affect the mouth, especially the tongue, floor of the mouth, lips, oral mucosa, soft palate, uvula, gingiva and alveolar mucosa as superficial ulcers, hard nodules in soft tissues or central bone lesions (tuberculous osteomyelitis). The disease is confirmed by sputum examination, Mantoux reaction, chest $x$-rays and biopsy of the lesion ${ }^{9,10,12}$.

In the present case, lesion type and location led to the suspicion of syphilis. This was ruled out by investigation of the patient's sexual life and a non-reactive VDRL test. Similarly, tuberculosis was raised as a diagnostic hypothesis, especially because of the characteristic of the lesion. This was ruled out based on chest radiography, which showed normal pulmonary fields and because the patient was in good general health.

Once syphilis and tuberculosis were ruled out in this case, only $P G$ remained as a diagnostic hypothesis. Initially, chemical irritation resulting from the use of a paint solvent for cauterization of the initial blister (possibly a blister resulting from the fusion of herpes vesicles frequently found at this site) was believed to be the etiological agent. This hypothesis was not supported since the patient reported use of the solvent only to control bleeding, which is uncommon for a herpes lesion. When asked, the patient denied the habit of biting on her upper lip, which made the determination of a traumatic etiological agent inconclusive.

In a 12-year old female patient, an age characterized by important hormonal changes and hormonal factors that can be related to the PG's etiology ${ }^{7}$, this lesion may be related to hormonal etiology.

Despite the possible occurrence of $\mathrm{scars}^{5}$, biopsy is the method for adequate diagnosis of oral lesions ${ }^{9}$ and surgical excision is the most common treatment of $\mathrm{PGs}^{12}$. In this case, an excisional biopsy was performed for diagnosis and treatment. The procedure was simple, essential for final diagnosis, and provided a rapid result to fulfill patient expectations. In the present case, there was a discrete scar and the patient was psychologically satisfied with the outcome.

\section{CONCLUSIONS}

This case reports emphasizes that the diagnosis of oral lesions is complex and leads the dentist to consider distinct lesions with different diagnostic methods. We call attention to the uncommon mucocutaneous labial location of PG and to the fact that surgical excision is the safest method for diagnosis and treatment of PG of the lip, even when involving the mucosa and skin.

\section{REFERENCES}

1- Al-Khateeb T, Ababneh K. Oral pyogenic granuloma in Jordanians: a retrospective analysis of 108 cases. J Oral Maxillofac Surg. 2003;61:1285-8.

2- Damm DD, Fantasia JE. Elevated and ulcerated nodule of lip. Pyogenic granuloma. Gen Dent. 2002;50:466-8.

3- Galeckas KJ, Uebelhoer NS. Successful treatment of pyogenic granuloma using a 1,064-nm laser followed by glycerin sclerotherapy. Dermatol Surg. 2009;35:530-4.

4- Graham RM. Pyogenic granuloma: an unusual presentation. Dent Update. 1996;23:240-1.

5- Ichimiya M, Yoshikawa Y, Hamamoto Y, Muto M. Successful treatment of pyogenic granuloma with injection of absolute ethanol. J Dermatol. 2004;31:342-4.

6- Ishida CE, Ramos-e-Silva M. Cryosurgery in oral lesions. Int J Dermatol. 1998;37:283-5.

7- Jafarzadeh $H$, Sanatkhani M, Mohtasham N. Oral pyogenic granuloma: a review. J Oral Sci. 2006;48:167-75.

8- Lin RL, Janniger CK. Pyogenic granuloma. Cutis. 2004;74:22933.

9- Neville BW, Damm DD, Allen CM. Patologia oral e maxilofacial. Rio de Janeiro: Guanabara Koogan; 1998.

10- Regezi JA, Sciuba JJ. Oral pathology, clinical pathological correlations. Philadelphia: Saunders; 1989.

11- Saravana GH. Oral pyogenic granuloma: a review of 137 cases. Brit J Oral Maxillofac Surg. 2009;47:318-9.

12- Shafer WG, Hine MK, Levy BM. A textbook of oral pathology. Philadelphia: Saunders; 1983.

13- Souza LN, Martins CR, Paula AM. Cutaneous horn occurring on the lip of a child. Int J Paediatr Dent. 2003;13:365-7.

14- Wang SQ, Goldberg LH. Treatment of recurrent pyogenic granuloma with excision and frozen section for margin control. Dermatol Surg. 2008;34:1115-6. 